\title{
Plasma ADMA, urinary ADMA excretion, and late mortality in renal transplant recipients
}

\author{
M. Yusof Said ${ }^{1}\left(\mathbb{D} \cdot\right.$ A. Bollenbach ${ }^{2}$. Isidor Minović ${ }^{3} \cdot$ Marco van Londen $^{1}$ - Anne-Roos Frenay ${ }^{4} \cdot$ Martin H. de Borst ${ }^{1,6}$. \\ Else van den Berg ${ }^{1}$ - A. Arinc Kayacelebi ${ }^{2} \cdot$ Dimitrios Tsikas $^{2} \cdot$ Harry van Goor ${ }^{5,6} \cdot$ Gerjan Navis $^{1,6}$. \\ Stephan J. L. Bakker ${ }^{1,6}$
}

Received: 24 June 2018 / Accepted: 12 March 2019 / Published online: 26 March 2019

(c) The Author(s) 2019

\begin{abstract}
Cardiovascular disease (CVD) is the leading cause of death in renal transplant recipients (RTR). Elevated plasma asymmetric dimethylarginine (pADMA), an endogenous nitric oxide synthase inhibitor produced from the turnover of methylated arginine moieties in proteins, is a risk factor for CVD and mortality. It is unknown how urinary ADMA excretion (uADMA), one of the main ADMA elimination routes, is associated with long-term survival. Furthermore, the association of pADMA and uADMA with markers for turnover of arginine-methylated proteins is unknown. We analyzed ADMA using a validated GC-MS/MS method in plasma and 24-h urine samples of 685 RTR, included $\geq 1$ year after transplantation. We also analyzed urine symmetric dimethylarginine (USDMA) using the same method. Urinary creatinine and urea excretions were used as markers for turnover of muscle protein and amino acids, respectively. We applied Cox regression analyses to study associations of pADMA, uADMA, and uSDMA with all-cause and CVD mortality. Mean pADMA was $0.61 \pm 0.12 \mu \mathrm{M}$, uADMA was $31 \pm 13 \mu \mathrm{mol} / 24 \mathrm{~h}$, and uSDMA was $52 \pm 19 \mu \mathrm{mol} / 24 \mathrm{~h}$. Over median follow-up of 5.4 [4.9-6.1] years, 147 RTR died, of which 58 (39\%) from CVD. High pADMA was associated with high all-cause mortality (HR per SD [95\% CI]: 1.45 [1.26-1.67], $P<0.001$ ), while high uADMA was associated with low all-cause and CVD mortality (HR per SD [95\% CI]: 0.57 [0.47-0.69], $P<0.001$, and 0.55 [0.40-0.74], $P<0.001$, respectively). The associations were independent of adjustment for potential confounders. Creatinine excretion was associated with both pADMA (st. $\beta:-0.21, P=0.003$ ) and uADMA (st. $\beta: 0.49, P<0.001$ ), and urea excretion was associated with uADMA (st. $\beta: 0.56, P<0.001$ ). Associations of uSDMA with outcomes and with creatinine excretion and urea excretion were comparable to those of uADMA. The associations of pADMA, uADMA and USDMA with mortality were strongly affected by adjustment for creatinine excretion and urea excretion. We found for the first time that high uADMA and high uSDMA are associated with less risk of all-cause and CVD mortality. The links of UADMA and USDMA with markers of muscle protein and amino acid turnover may serve to further understand ADMA and SDMA homeostasis and their clinical implications.
\end{abstract}

Keywords Kidney transplantation $\cdot$ ADMA $\cdot$ SDMA $\cdot$ Muscle mass $\cdot$ Protein turnover $\cdot$ Long-term survival

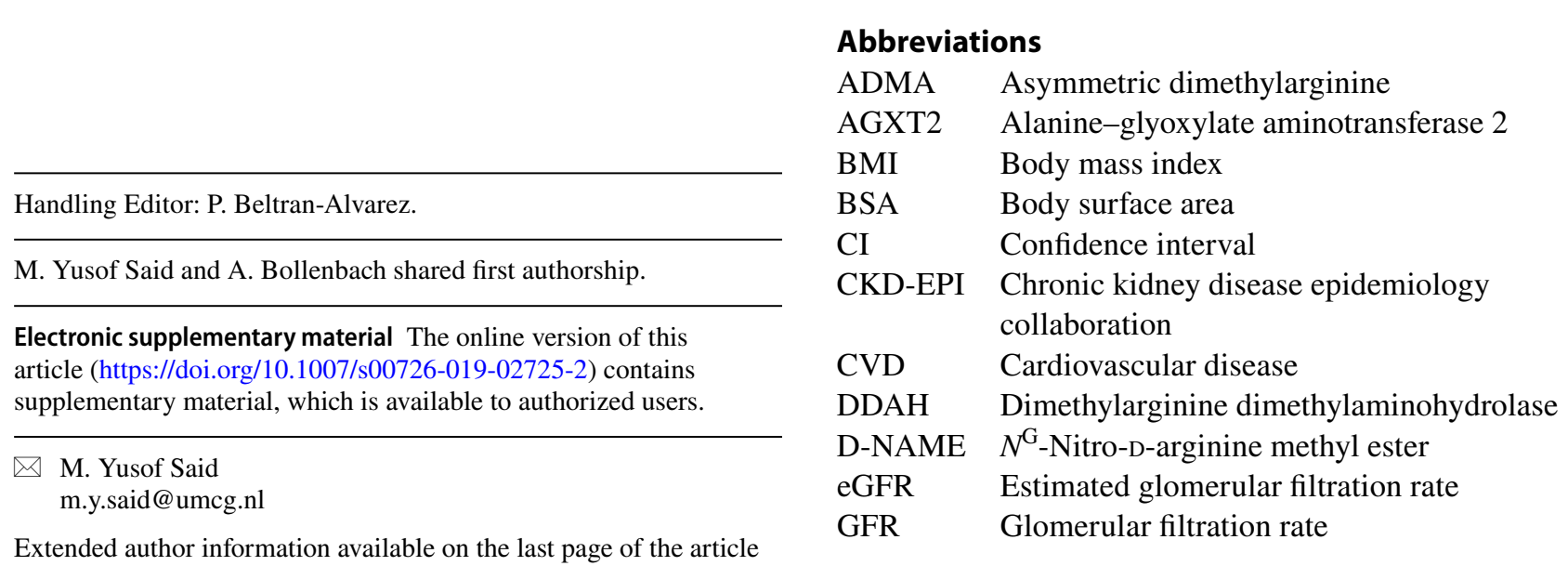




$\begin{array}{ll}\text { HR } & \text { Hazard ratio } \\ \text { IQR } & \text { Interquartile range } \\ \text { L-NAME } & N^{\mathrm{G}} \text {-Nitro-L-arginine methyl ester } \\ \text { mGFR } & \text { Measured glomerular filtration rate } \\ \text { MMA } & \text { Monomethylated arginine } \\ \text { mTOR } & \text { Mammalian target of rapamycin } \\ \text { NOS } & \text { Nitric oxide synthase } \\ \text { pADMA } & \text { Plasma asymmetric dimethylarginine } \\ \text { PRMT } & \text { Protein arginine } N \text {-methyltransferase } \\ \text { RTR } & \text { Renal transplant recipient } \\ \text { SDMA } & \text { Symmetric dimethylarginine } \\ \text { uADMA } & \text { Urinary asymmetric dimethylarginine } \\ \text { uSDMA } & \text { Urinary symmetric dimethylarginine }\end{array}$

\section{Introduction}

Cardiovascular disease (CVD) is the leading cause of death in renal transplant recipients (RTR). With the increasing number of transplantations and people living with a renal transplant (Saran et al. 2018), it is of particular importance to find population-specific risk factors for cardiovascular disease and mortality. Asymmetric dimethylarginine (ADMA) is an endogenous nitric oxide synthase (NOS) inhibitor and produced from the turnover of arginine-methylated proteins (Teerlink 2005). High plasma ADMA (pADMA) is considered a risk factor for premature mortality and cardiovascular disease in the general population, patients with chronic kidney disease, and RTR (Zoccali et al. 2001, 2002; Achan et al. 2003; Wolf et al. 2012; Frenay et al. 2015). Since renal excretion of ADMA is one of the main ways of its elimination and PADMA concentrations increase with worse renal function, ADMA is considered a uremic toxin (Kielstein and Zoccali 2005; Jacobi and Tsao 2008). Urinary ADMA excretion (UADMA) is, therefore, an interesting, yet understudied component of ADMA homeostasis. In RTR, it is unknown how UADMA is associated with long-term outcomes and how pADMA and UADMA influence each other's association with long-term outcomes. Furthermore, it is unknown how ADMA is associated with markers for its production from the turnover of arginine-methylated protein sources.

We aimed to study pADMA and 24-h uADMA, and their potential associations with all-cause and cardiovascular mortality in a large prospective cohort of stable RTR after transplantation. Second, in a sub-population, we aimed to study the association of pADMA and UADMA with markers of protein turnover: urinary creatinine excretion and urinary urea excretion, reflecting turnover of muscle and amino acids, respectively (Maroni et al. 1985; Wyss and KaddurahDaouk 2000), while adjusting for differences in true glomerular filtration rate (GFR). In addition to our analyses for the urinary excretion of ADMA, we performed analyses for the urinary excretion of symmetric dimethylarginine
(uSDMA). Symmetric dimethylarginine (SDMA) is an isomer of ADMA which does not inhibit NOS and is eliminated almost exclusively via the kidneys (Nijveldt et al. 2002).

\section{Methods and materials}

\section{Study population}

Adult RTR ( $n=817)$ who were treated at the University Medical Center Groningen, The Netherlands, were invited to participate in the study between November 2008 and March 2011. The current study is part of a larger prospective cohort study of RTR in the North of the Netherlands (Transplantlines Food and Nutrition cohort, Clinicaltrials. gov No NCT02811835). Inclusion criteria were a renal transplantation $\geq 1$ year before inclusion and a functioning graft. Exclusion criteria were a history of alcohol or drug abuse, malignancy (other than cured skin cancer), overt congestive heart failure (NYHA 3-4), and insufficient understanding of Dutch. Out of 817 RTR invited to participate, 706 RTR signed a written informed consent. After excluding those without data on pADMA and uADMA, 685 RTR were included in the present study. Data on uSDMA were available in 673 RTR. Subjects were at steady state at all measurements, i.e., without acute illness and biochemically stable. The study was approved by the institutional ethical review board (METc 2008/186) and has been conducted in accordance with the declaration of Helsinki.

\section{Clinical measurements}

Blood was drawn after a fasting period of at least $8 \mathrm{~h}$. Each participant collected 24-h urine according to instructions. Detailed descriptions of anthropomorphic measurements have been described before (van den Berg et al. 2012, 2013, 2014). We performed the anthropomorphic measurements on the same day as blood collection. Body surface area (BSA) was calculated using the Du Bois-Du Bois formula (Du Bois and Du Bois 1916). Body mass index (BMI) was calculated as weight $(\mathrm{kg})$ divided by squared height (meters). Smoking status and alcohol intake were measured by questionnaire. We categorized smoking as never, ex, or current. Alcohol intake was categorized as $0-10,10-30$, or $>30 \mathrm{~g} /$ day.

\section{Laboratory measurements}

We measured pADMA, uADMA, and USDMA with a validated GC-MS/MS method (Thermoquest TSQ 7000, Finnigan MAT, San Jose, California, USA) as described in detail earlier (Tsikas et al. 2003; Frenay et al. 2015; Bollenbach et al. 2018). Other urine and blood analyses have been 
performed using routine laboratory methods as described earlier (van den Berg et al. 2012, 2013, 2014).

We calculated the estimated glomerular filtration rate (eGFR) using the Chronic Kidney Disease Epidemiology Collaboration (CKD-EPI) formula with serum creatinine and cystatin C. In a subset of the study population $(n=201)$, true GFR (measured GFR; mGFR) was measured by infusion of low-dose ${ }^{125}$ I-iothalamate, as previously described (Apperloo et al. 1996). We defined proteinuria as a urinary excretion of $\geq 0.5 \mathrm{~g} / 24 \mathrm{~h}$.

\section{Long-term outcomes}

Long-term outcomes were all-cause mortality, CVD mortality, and non-cardiovascular disease (non-CVD) mortality. CVD mortality was defined as death as a result of cardiovascular disease (ICD-9: 410-447): coronary artery disease, cerebrovascular accident, heart failure, and sudden death, as described before (National Center for Health Statistics 2011; Minović et al. 2017). The cause of death was retrieved from medical records or, if necessary, from correspondence with the RTR's general practitioner or referring hospital.

\section{Statistical analyses}

We performed univariable linear regression analyses of pADMA, uADMA, and uSDMA with all basic characteristics to study those which are associated with pADMA, uADMA, or uSDMA. Kaplan-Meier analyses were performed for the associations of sex-adjusted tertiles of pADMA, uADMA, and uSDMA with outcomes and differences between tertiles tested by log-rank tests. We applied multivariable Cox regression analyses to study the associations of pADMA, uADMA, and uSDMA with outcomes. The proportionality of hazards assumption was tested with the Schoenfield residual test and was not violated for the associations of pADMA, uADMA, or uSDMA with all outcomes ( $P>0.05$ for all analyses). To test the assumption for linearity, we applied restricted cubic splines (knots: minimum, median, and maximum) for non-transformed variables and compared these with linear splines. Splines were fitted on a Cox model and adjusted for age, sex, BMI, eGFR, and proteinuria. We used two-sided Wald test for non-linearity analysis: $P$ for non-linearity of the association of pADMA, uADMA, and USDMA with all-cause, CVD, and non-CVD mortality was for all $>0.05$. Partially, cumulative models were used to reduce the possibility of over-fitting for the Cox regression analyses: we adjusted for basic confounders (age, sex, BMI, eGFR, proteinuria) in model 1 . We added other potential confounders to model 1 in all subsequent models. In the model 5, we adjusted the associations of pADMA with outcomes for UADMA (model 5a) and uSDMA (model $5 b$ ), the associations of UADMA for pADMA (model 5) and
uSDMA (model 5b), and the associations of uSDMA for pADMA (model 5) and uADMA (model 5a). In the final model, we adjusted the associations of pADMA, uADMA, and USDMA for each other in a combined model. We performed secondary analyses in a smaller subset of the study population $(n=201)$ who had data available on mGFR by low-dosage ${ }^{125}$ I-iothalamate, as described earlier (Apperloo et al. 1996), to study the association of muscle mass turnover, reflected by 24 -h urinary creatinine excretion, and amino acid turnover, reflected by 24-h urinary urea excretion, with pADMA, uADMA, and uSDMA. We also studied the effects of adjusting for urinary creatinine excretion and urea excretion in the associations of pADMA, UADMA, and uSDMA with mortality.

We tested for the interactions of pADMA, uADMA, uSDMA with each other, age, sex, BMI, eGFR, proteinuria, urinary creatinine excretion, and urinary urea excretion by adding an interaction-variable composed of the product of pADMA, uADMA, or uSDMA and the above-mentioned variables.

We performed analyses with IBM SPSS statistics version 23 (2015, IBM corp., Armonk, NY, USA). Figures were made with GraphPad Prism version 5.04 for Windows (2010, GraphPad Software, La Jolla, California, USA) and R statistics version 3.2.3 (2015, R Foundation for Statistical Computing, Vienna, Austria). Normally distributed variables are presented as mean \pm standard deviation (SD), skewed data as median [interquartile range (IQR)]. We regarded a $P$ value of $\leq 0.05$ as statistically significant.

\section{Results}

Basic characteristics of 685 RTR are presented in Table 1. pADMA is $0.61 \pm 0.12 \mu \mathrm{M}$, uADMA is $31.4 \pm 13.2 \mu \mathrm{mol} / 24 \mathrm{~h}$, and uSDMA is $52.3 \pm 19.1 \mu \mathrm{mol} / 24 \mathrm{~h}$. The Pearson correlation between pADMA and uADMA is $-0.08, P=0.04$. Pearson correlation of pADMA and uSDMA is $-0.09, P=0.02$. Pearson correlation of UADMA with uSDMA is 0.53 , $P<0.001$. High pADMA, uADMA, and USDMA are associated with the male gender. High uADMA shows an opposite association pattern compared to pADMA with regard to its associations with less use of antihypertensive drugs, less history of CVD, more physical activity, higher urinary creatinine excretion, higher urinary urea excretion, living donor transplantation, shorter cold ischemia time, immunosuppressive medication usage, and overall better renal function. Higher UADMA is also associated with larger weight, higher BMI and BSA, lower total and low-density lipoprotein cholesterol, lower triglycerides, lower $\mathrm{C}$-reactive protein, lower $\mathrm{N}$-terminal pro-brain natriuretic peptide, less diabetes as primary disease cause, 
Table 1 Basic characteristics

\begin{tabular}{|c|c|c|c|c|c|c|c|}
\hline & \multirow[t]{2}{*}{ RTR } & \multicolumn{2}{|c|}{$\begin{array}{l}\text { Association with } \\
\text { plasma ADMA }\end{array}$} & \multicolumn{2}{|c|}{$\begin{array}{l}\text { Association with } \\
\text { urinary ADMA excre- } \\
\text { tion }\end{array}$} & \multicolumn{2}{|c|}{$\begin{array}{l}\text { Association with } \\
\text { urinary SDMA } \\
\text { excretion }\end{array}$} \\
\hline & & St. $\beta$ & $P$ & St. $\beta$ & $P$ & St. $\beta$ & $P$ \\
\hline Number of participants & 685 & 685 & & 685 & & 673 & \\
\hline \multicolumn{8}{|l|}{ ADMA } \\
\hline Plasma ADMA $(\mu \mathrm{M})$ & $0.61 \pm 0.12$ & N/A & & -0.08 & 0.04 & -0.09 & 0.02 \\
\hline Urinary ADMA excretion $(\mu \mathrm{mol} / 24 \mathrm{~h})$ & $31.4 \pm 13.2$ & -0.08 & 0.04 & N/A & & 0.53 & $<0.001$ \\
\hline Urinary SDMA excretion, $(\mu \mathrm{mol} / 24 \mathrm{~h})$ & $52.3 \pm 19.1$ & -0.09 & 0.02 & 0.53 & $<0.001$ & N/A & \\
\hline \multicolumn{8}{|l|}{ Demographics } \\
\hline Age of patient (years) & $54.7[44.8-63.0]$ & 0.12 & 0.001 & -0.05 & 0.16 & -0.07 & 0.08 \\
\hline Male gender, $n(\%)$ & $390(56.9)$ & $\mathbf{0 . 0 8}$ & $\mathbf{0 . 0 3}$ & 0.23 & $<0.001$ & 0.30 & $<0.001$ \\
\hline \multicolumn{8}{|l|}{ Body composition (recipient) } \\
\hline Weight $(\mathrm{kg})$ & $80.4 \pm 16.4$ & -0.05 & 0.24 & 0.22 & $<0.001$ & 0.25 & $<0.001$ \\
\hline $\mathrm{BMI}\left(\mathrm{kg} / \mathrm{m}^{2}\right)$ & $26.0[23.2-29.3]$ & -0.05 & 0.23 & 0.12 & 0.002 & 0.08 & 0.04 \\
\hline $\operatorname{BSA}\left(\mathrm{m}^{2}\right)$ & $1.94 \pm 0.22$ & -0.05 & 0.23 & 0.25 & $<0.001$ & 0.30 & $<0.001$ \\
\hline \multicolumn{8}{|l|}{ Cardiovascular parameters } \\
\hline Systolic blood pressure (mmHg) & $136 \pm 17.4$ & 0.03 & 0.50 & -0.02 & 0.67 & 0.06 & 0.16 \\
\hline Diastolic blood pressure (mmHg) & $82.5 \pm 10.9$ & -0.04 & 0.33 & 0.06 & 0.11 & 0.11 & 0.01 \\
\hline \multicolumn{8}{|l|}{ Number of antihypertensives, $n(\%)^{\mathrm{c}}$} \\
\hline 0 antihypertensive drugs & $79(11.5)$ & Ref & & Ref & & & \\
\hline 1 antihypertensive drugs & $108(27.4)$ & 0.08 & 0.21 & -0.07 & 0.21 & -0.02 & 0.79 \\
\hline$\geq 2$ antihypertensive drugs & $418(61.0)$ & 0.15 & 0.01 & -0.20 & 0.001 & -0.03 & 0.61 \\
\hline NTpro-BNP (ng/L) & $250[106-625]$ & 0.07 & 0.09 & -0.19 & $<0.001$ & -0.12 & 0.003 \\
\hline \multicolumn{8}{|l|}{ Cardiovascular disease history } \\
\hline Myocardial infarction, $n(\%)$ & $34(5.0)$ & 0.08 & $\mathbf{0 . 0 3}$ & 0.01 & 0.76 & -0.003 & 0.94 \\
\hline CABG and/or PCI, $n(\%)$ & $55(8.0)$ & 0.10 & 0.01 & -0.05 & 0.19 & -0.02 & 0.53 \\
\hline CVA and/or TIA, $n(\%)$ & $39(5.7)$ & -0.04 & 0.31 & -0.08 & 0.04 & -0.03 & 0.41 \\
\hline \multicolumn{8}{|l|}{ Diabetes } \\
\hline Diabetes $n(\%)^{\mathrm{a}}$ & $162(23.6)$ & 0.08 & 0.05 & -0.04 & 0.29 & -0.04 & 0.29 \\
\hline Glucose (mM) & $5.2[4.8-6.0]$ & 0.06 & 0.15 & 0.02 & 0.57 & -0.04 & 0.36 \\
\hline $\operatorname{HbA1c}(\%)$ & $5.8[5.5-6.2]$ & 0.02 & 0.71 & 0.03 & 0.48 & -0.03 & 0.40 \\
\hline \multicolumn{8}{|l|}{ Lipids } \\
\hline Total cholesterol (mM) & $5.13 \pm 1.13$ & -0.05 & 0.17 & -0.14 & $<0.001$ & -0.15 & $<0.001$ \\
\hline HDL cholesterol (mM) & $1.30[1.10-1.60]$ & -0.14 & $<0.001$ & 0.02 & 0.62 & -0.06 & 0.13 \\
\hline LDL cholesterol (mM) & $2.90[2.30-3.50]$ & -0.02 & 0.54 & -0.08 & 0.04 & -0.10 & 0.01 \\
\hline Triglycerides (mM) & $1.68[1.25-2.30]$ & 0.02 & 0.59 & -0.17 & $<0.001$ & -0.11 & 0.004 \\
\hline \multicolumn{8}{|l|}{ Inflammation } \\
\hline $\mathrm{CRP}(\mathrm{mg} / \mathrm{L})$ & $1.6[0.7-4.6]$ & -0.03 & 0.47 & -0.08 & 0.04 & -0.06 & 0.12 \\
\hline Blood leukocyte $\left(\times 10^{9} / \mathrm{L}\right)$ & $8.1 \pm 2.6$ & -0.08 & 0.03 & 0.04 & 0.31 & 0.01 & 0.74 \\
\hline \multicolumn{8}{|l|}{ Smoking behavior, $n(\%)^{\mathrm{b}}$} \\
\hline Never & $269(39.3)$ & Ref. & & Ref. & & & \\
\hline Ex & $293(42.8)$ & 0.01 & 0.79 & -0.02 & 0.57 & -0.01 & 0.88 \\
\hline Current & $82(12.0)$ & -0.01 & 0.88 & 0.01 & 0.77 & 0.03 & 0.51 \\
\hline \multicolumn{8}{|l|}{ Alcohol intake, $n(\%)^{\mathrm{b}}$} \\
\hline $0-10 \mathrm{~g} / 24 \mathrm{~h}$ & $459(67.0)$ & Ref. & & Ref & & & \\
\hline $10-30 \mathrm{~g} / 24 \mathrm{~h}$ & $136(19.9)$ & -0.01 & 0.81 & 0.04 & 0.30 & 0.15 & $<0.001$ \\
\hline$>30 \mathrm{~g} / 24 \mathrm{~h}$ & $29(4.2)$ & -0.02 & 0.59 & 0.06 & 0.13 & 0.13 & 0.001 \\
\hline Physical activity (SQUASH score) & $5070[1970-8018]$ & -0.10 & 0.01 & 0.09 & 0.02 & 0.13 & 0.001 \\
\hline Urinary creatinine excretion (mmol/24 h) & $11.6 \pm 3.5$ & -0.16 & $<0.001$ & 0.50 & $<0.001$ & 0.54 & $<0.001$ \\
\hline Urinary urea excretion (mmol/24 h) & $388 \pm 115$ & -0.09 & 0.02 & 0.51 & $<0.001$ & 0.52 & $<0.001$ \\
\hline
\end{tabular}


Table 1 (continued)

\begin{tabular}{|c|c|c|c|c|c|c|c|}
\hline & \multirow[t]{2}{*}{ RTR } & \multicolumn{2}{|c|}{$\begin{array}{l}\text { Association with } \\
\text { plasma ADMA }\end{array}$} & \multicolumn{2}{|c|}{$\begin{array}{l}\text { Association with } \\
\text { urinary ADMA excre- } \\
\text { tion }\end{array}$} & \multicolumn{2}{|c|}{$\begin{array}{l}\text { Association with } \\
\text { urinary SDMA } \\
\text { excretion }\end{array}$} \\
\hline & & St. $\beta$ & $P$ & St. $\beta$ & $P$ & St. $\beta$ & $P$ \\
\hline \multicolumn{8}{|l|}{ Pre-transplant disease, $n(\%)^{\mathrm{c}}$} \\
\hline Dysplasia and hypoplasia & $28(4.1)$ & 0.01 & 0.78 & 0.02 & 0.71 & 0.05 & 0.23 \\
\hline Glomerulonephritis & $51(7.4)$ & -0.04 & 0.32 & -0.03 & 0.56 & 0.04 & 0.41 \\
\hline Diabetes mellitus & $34(5.0)$ & 0.05 & 0.21 & -0.12 & 0.01 & -0.03 & 0.55 \\
\hline Polycystic renal disease & $142(20.7)$ & 0.04 & 0.44 & -0.07 & 0.19 & 0.03 & 0.55 \\
\hline Primary glomerular disease & $196(28.6)$ & -0.03 & 0.58 & -0.04 & 0.50 & 0.10 & 0.07 \\
\hline Renovascular disease & $39(5.7)$ & -0.004 & 0.93 & -0.06 & 0.19 & 0.09 & 0.04 \\
\hline Tubular interstitial disease & 79 (11.5) & -0.04 & 0.35 & -0.01 & 0.80 & 0.02 & 0.67 \\
\hline Other/unknown cause & $116(16.9)$ & Ref. & & Ref & & Ref & \\
\hline Time between transplantation and baseline (years) & $5.39[1.84-12.11]$ & 0.04 & 0.25 & -0.10 & 0.01 & -0.12 & 0.002 \\
\hline Total dialysis time (months) & $27[9-51]$ & 0.13 & 0.001 & -0.03 & 0.50 & $-\mathbf{0 . 0 8}$ & 0.05 \\
\hline Living donor transplantation, $n(\%)$ & $233(34.0)$ & -0.12 & 0.002 & 0.09 & 0.02 & 0.11 & 0.01 \\
\hline \multicolumn{8}{|l|}{ Ischemia times } \\
\hline Cold ischemia times (h) & $15.4[2.8-21.2]$ & 0.11 & 0.004 & -0.11 & 0.004 & -0.10 & 0.01 \\
\hline Warm ischemia times (min) & $40[34-50]$ & 0.02 & 0.69 & 0.03 & 0.48 & 0.01 & 0.80 \\
\hline Rejection before baseline, $n(\%)$ & $181(26.4)$ & 0.02 & 0.64 & -0.12 & 0.001 & -0.02 & 0.63 \\
\hline \multicolumn{8}{|l|}{ Number of transplantations, $n(\%)$} \\
\hline 1 & $616(89.9)$ & Ref. & & Ref & & & \\
\hline 2 or more & $69(10.1)$ & 0.07 & 0.09 & -0.09 & 0.02 & -0.10 & 0.01 \\
\hline \multicolumn{8}{|l|}{ Immunosuppressive medication } \\
\hline Prednisolone dosage $(\mathrm{mg} / 24 \mathrm{~h})$ & $10.0[7.5-10.0]$ & 0.06 & 0.15 & 0.10 & 0.01 & 0.11 & 0.003 \\
\hline CNI usage $n(\%)$ & $390(56.9)$ & 0.09 & 0.01 & -0.15 & $<0.001$ & 0.03 & 0.51 \\
\hline Tacrolimus & $119(17.4)$ & $0.09^{\mathrm{e}}$ & $\mathbf{0 . 0 3}$ & $-0.17^{\mathrm{e}}$ & $<0.001$ & $-0.002^{\mathrm{e}}$ & 0.96 \\
\hline Cyclosporine A & $272(39.7)$ & $\mathbf{0 . 0 8}$ & 0.05 & $-0.12^{\mathrm{e}}$ & 0.002 & $0.03^{\mathrm{e}}$ & 0.44 \\
\hline Proliferation inhibitor usage $n(\%)$ & $571(83.4)$ & -0.10 & 0.01 & 0.09 & 0.02 & 0.06 & 0.12 \\
\hline Mycophenolate mofetil & $451(65.8)$ & $-0.14^{\mathrm{e}}$ & 0.004 & $0.15^{\mathrm{e}}$ & 0.002 & $0.11^{\mathrm{e}}$ & 0.03 \\
\hline Azathioprine & $120(17.5)$ & $-0.06^{\mathrm{e}}$ & 0.27 & $-0.02^{\mathrm{e}}$ & 0.72 & $-0.04^{\mathrm{e}}$ & 0.47 \\
\hline mTOR inhibitor usage ${ }^{\mathrm{d}}$ & $20(2.9)$ & -0.08 & 0.04 & 0.02 & 0.55 & -0.06 & 0.11 \\
\hline \multicolumn{8}{|l|}{ Renal allograft function } \\
\hline Serum urea $(\mathrm{mM})$ & $9.6[7.2-13.4]$ & 0.23 & $<0.001$ & -0.65 & $<0.001$ & -0.23 & $<0.001$ \\
\hline Serum creatinine $(\mu \mathrm{M})$ & 125 [99-162] & 0.15 & $<0.001$ & -0.57 & $<0.001$ & -0.14 & $<0.001$ \\
\hline $\mathrm{eGFR}^{\mathrm{f}}\left(\mathrm{mL} / \mathrm{min} / 1.73 \mathrm{~m}^{2}\right)$ & $43.5[30.6-58.0]$ & -0.31 & $<0.001$ & 0.72 & $<0.001$ & 0.25 & $<0.001$ \\
\hline $\mathrm{mGFR}^{\mathrm{g}}(\mathrm{mL} / \mathrm{min})$ & $55.1 \pm 20.5$ & -0.30 & $<0.001$ & 0.77 & $<0.001$ & 0.32 & $<0.001$ \\
\hline Protein excretion $(\mathrm{g} / 24 \mathrm{~h})$ & $0.19[0.02-0.37]$ & 0.03 & 0.41 & -0.15 & $<0.001$ & -0.004 & 0.92 \\
\hline Proteinuria ( $\geq 0.5 \mathrm{~g}$ per $24 \mathrm{~h}), n(\%)$ & $155(22.6)$ & 0.06 & 0.12 & -0.17 & $<0.001$ & -0.01 & 0.90 \\
\hline
\end{tabular}

$A D M A$ asymmetric dimethylarginine, SDMA symmetric dimethylarginine, $B M I$ body mass index, $B S A$ body surface area, $N T$-proBNP N-terminal pro-brain natriuretic peptide, $C A B G$ coronary artery bypass grafting, $P C I$ percutaneous coronary intervention, $C V A$ cerebrovascular accident, $T I A$ transient ischemic attack, $H D L$ high-density lipoprotein, $L D L$ low-density lipoprotein, $C R P$ C-reactive protein, $S Q U A S H$ short questionnaire to assess health enhancing physical activity, $C N I$ calcineurin inhibitor, $m T O R$ mammalian target of rapamycin, $e G F R$ estimated glomerular filtration rate, $m G F R$ measured glomerular filtration rate

${ }^{a}$ Defined as blood glucose $\geq 7 \mathrm{mM}, \mathrm{HbA} 1 \mathrm{c} \geq 6.5$, and/or use of antidiabetics

${ }^{\mathrm{b}}$ Percentages do not add up to $100 \%$ due to missing cases

${ }^{c}$ Percentages do not add up to $100 \%$ because of rounding

${ }^{\mathrm{d}}$ Sirolimus or everolimus

${ }^{\mathrm{e}}$ Reference is non-user

${ }^{\mathrm{f}}$ Chronic kidney disease epidemiology collaboration (CKD-EPI) formula with creatinine-cystatin C

${ }^{\mathrm{g}}$ Measured only in a smaller subset of 201 RTR 
shorter time between transplantation and baseline, less rejection before baseline, and lower number of previous transplantations. Higher pADMA is additionally associated with older age, lower blood leukocyte count, lower high-density lipoprotein cholesterol, more cases of diabetes mellitus, and longer dialysis time. uSDMA shows an association pattern comparable to that of uADMA, except for alcohol intake with which uSDMA is associated and uADMA not, and calcineurin inhibitor use with which uSDMA is not associated, while uADMA is.

\section{Associations with mortality}

Out of 685 RTR, 147 died during median follow-up of 5.4 [4.9-6.1] years. Of these, 58 (39\%) died of CVD. There were more all-cause and non-CVD mortalities in the highest sexadjusted tertile of pADMA compared to the lower tertiles (Fig. 1). In contrast, there were less all-cause, CVD, and non-CVD mortalities in the highest sex-adjusted tertile of uADMA and uSDMA compared to lower tertiles (Fig. 1). Figure 2 illustrates linear splines in which the distribution of pADMA, uADMA, and uSDMA is plotted against the hazard ratio of the respective variables for their associations with all-cause mortality, CVD mortality, and non-CVD
All-cause mortality

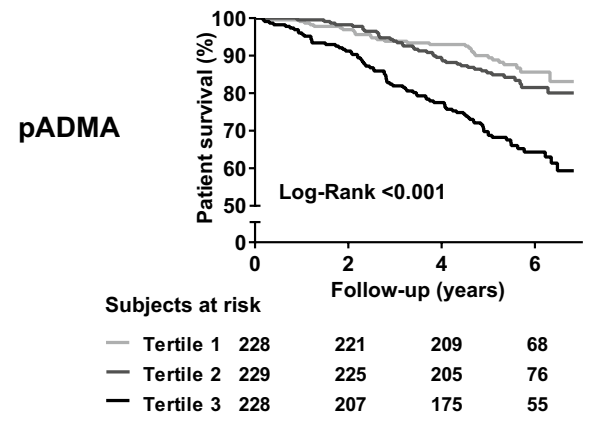

UADMA

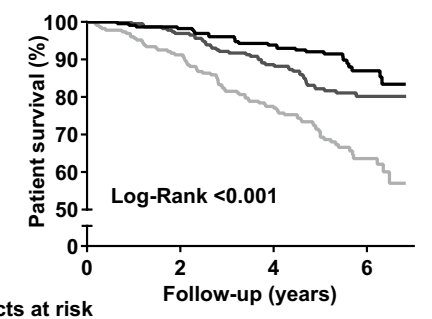

Subjects at risk

$\begin{array}{rrrrr}\text { - Tertile 1 } & 228 & 207 & 175 & 51 \\ \text { - Tertile 2 } & 229 & 222 & 201 & 73 \\ \text { - Tertile 3 } & 228 & 224 & 213 & 75\end{array}$

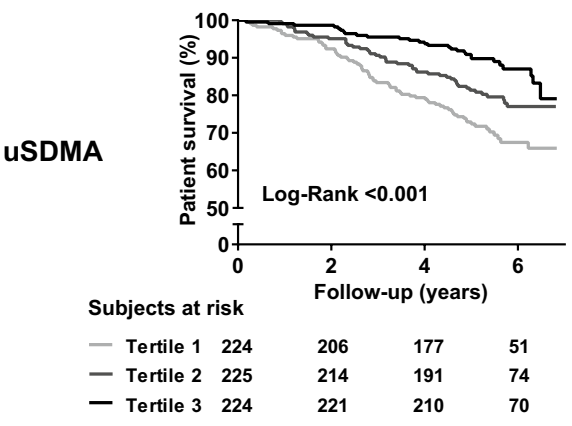

CVD mortality
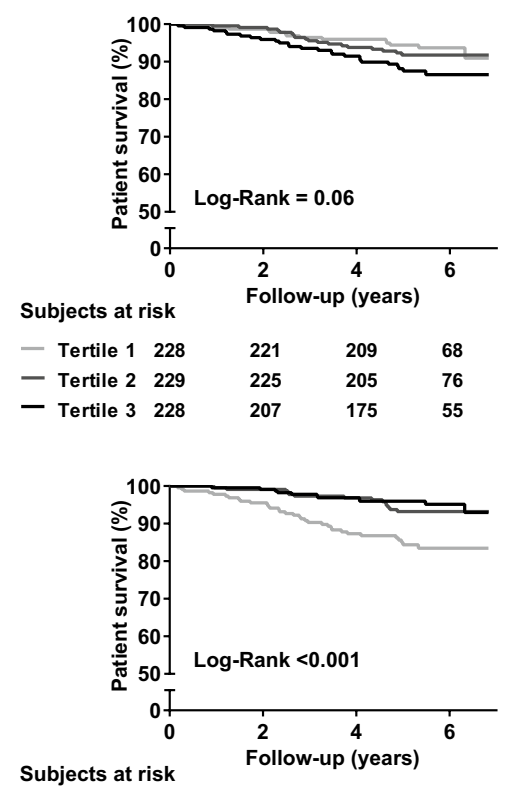

Subjects at risk

$$
\text { - Tertile } 1228
$$$$
\text { - Tertile } 2229
$$$$
\text { - Tertile } 3228
$$

$\begin{array}{lll}207 & 175 & 51 \\ 222 & 201 & 73 \\ 224 & 213 & 75\end{array}$

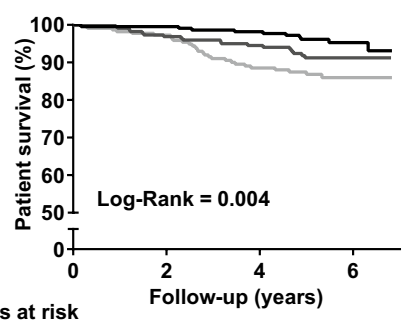

Subjects at risk

- Tertile 1224

- Tertile 2225 - Tertile 3224
Non-CVD mortality
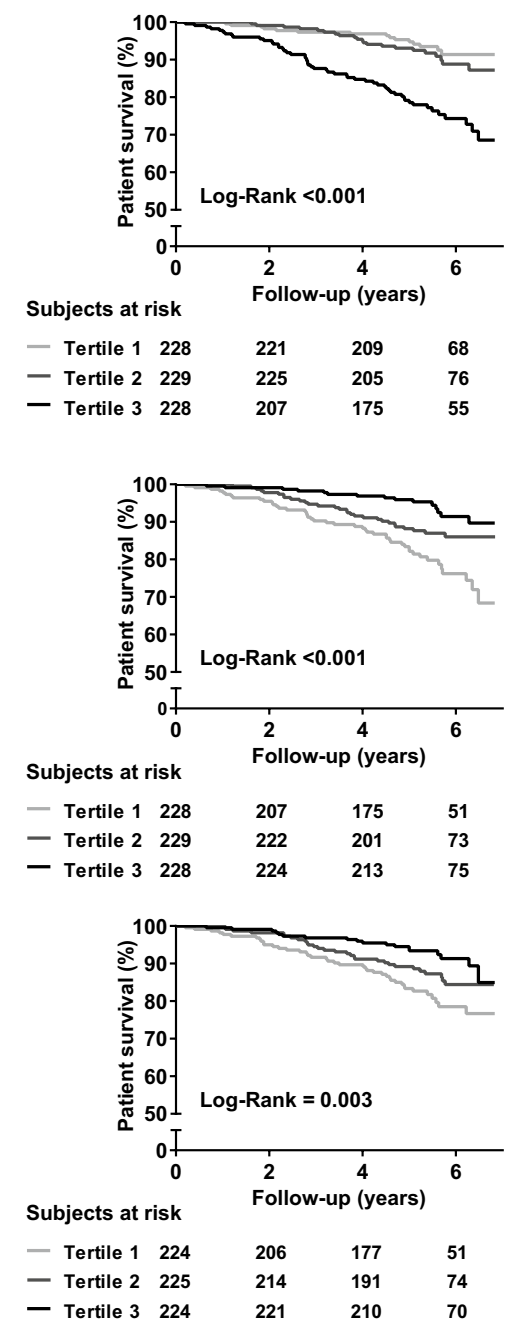

Fig. 1 Associations of sex-adjusted tertiles of plasma ADMA (pADMA), urinary ADMA excretion (uADMA), and urinary SDMA excretion (uSDMA) with all-cause mortality, cardiovascular (CVD) mortality, and non-cardiovascular (non-CVD) mortality. Median [range] in $\mu \mathrm{M}$ per tertile of pADMA: tertile 1: 0.50 [0.31-0.57]; ter- tile 2: 0.61 [0.54-0.66]; tertile 3: 0.72 [0.64-1.12]. Median [range] in $\mu \mathrm{mol} / 24 \mathrm{~h}$ per tertile of uADMA: tertile $1: 18.8$ [3.1-26.9]; tertile 2: 30.2 [22.0-39.1]; tertile 3: 43.7 [32.2-97.4]. Median [range] in umol/24 h per tertile of uSDMA: tertile 1: 35.3 [7.08-48.7]; tertile 2: 50.3 [38.5-63.5]; tertile 3: 67.9 [49.8-191.6] 
All-cause mortality
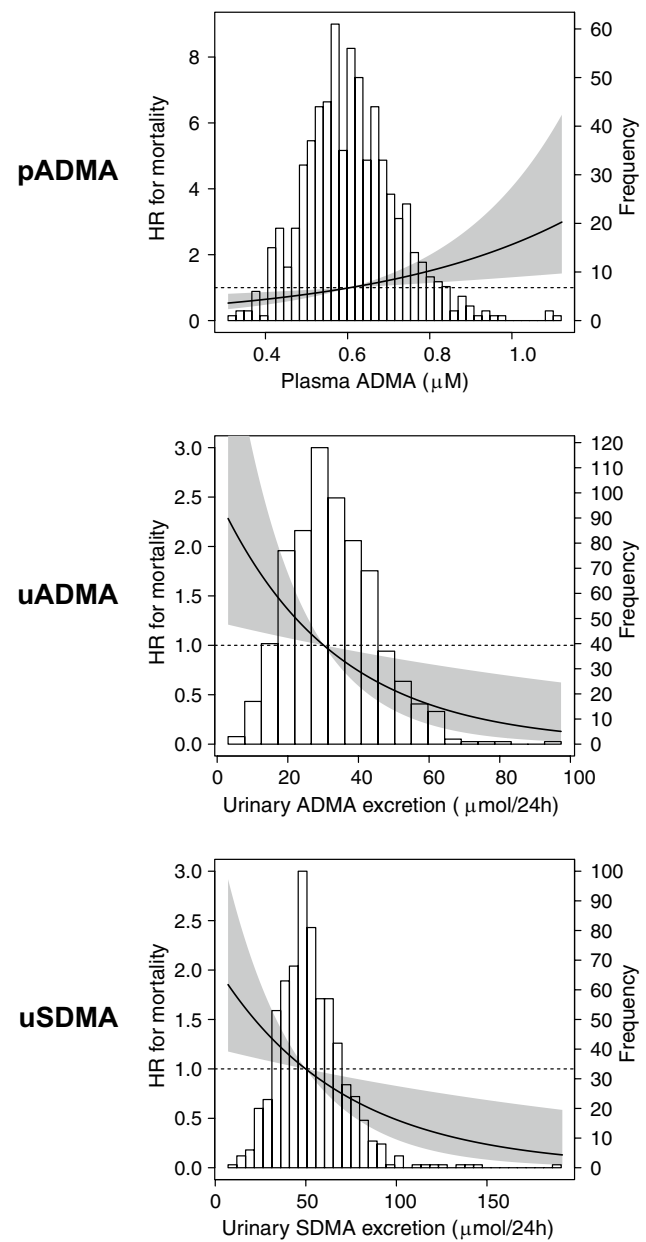

CVD-mortality
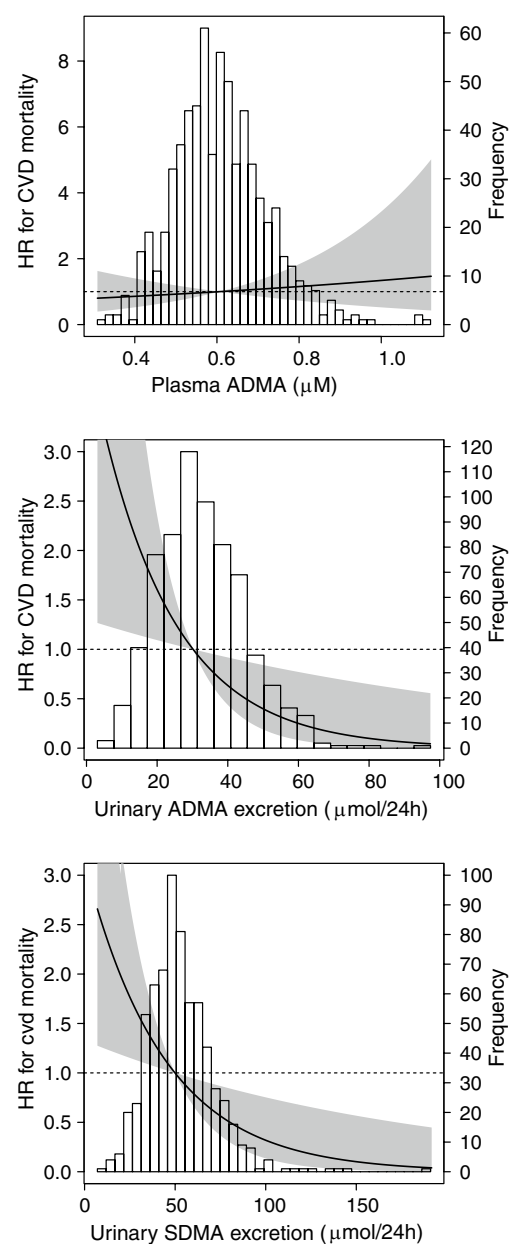

Non-CVD mortality
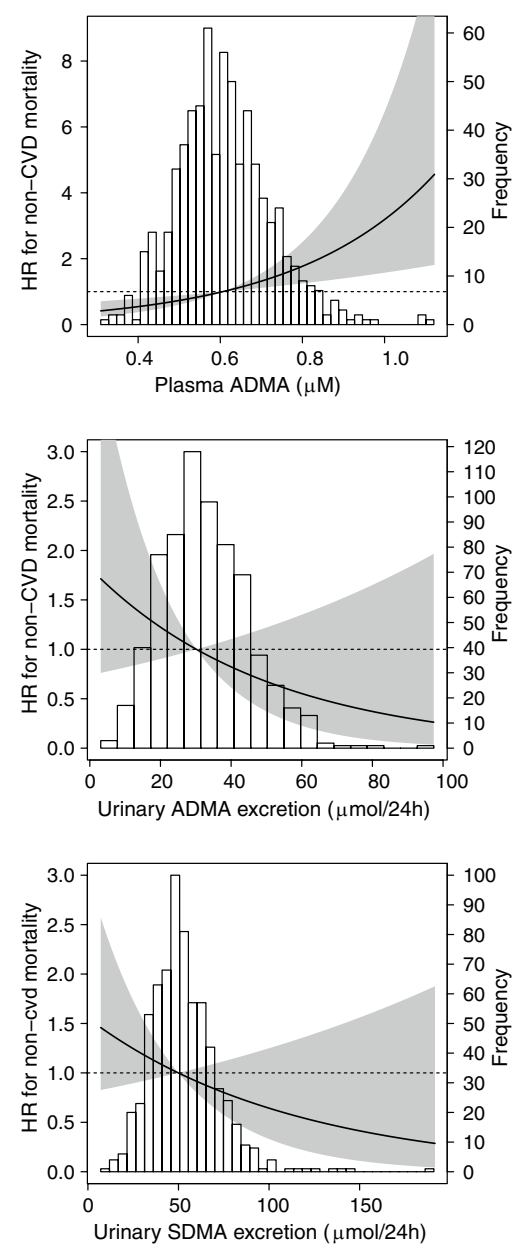

Fig. 2 Linear splines of the associations of plasma ADMA (pADMA), urinary ADMA excretion (uADMA), and urinary SDMA excretion (uSDMA) with all-cause mortality, cardiovascular disease (CVD) mortality, and non-CVD mortality. Data were fit by a Cox proportional hazard model for all splines and adjusted for age, sex, body mass index, proteinuria, and estimated glomerular filtration rate (chronic kidney disease epidemiology collaboration formula with creatinine-cystatin $\mathrm{C}$ ). The hazard ratio is represented by the black line, the $95 \%$ confidence interval by the gray area mortality (fitted by a Cox regression model, adjusted for age, sex, BMI, eGFR, and proteinuria).

In Table 2, Cox regression analyses of pADMA, uADMA, and USDMA with outcomes are presented. Univariably, pADMA is strongly associated with all-cause mortality (HR per SD [95\% CI]: 1.45 [1.26-1.67], $P<0.001)$, CVD mortality (HR per SD [95\% CI]: 1.30 [1.03-1.65], $P=0.03$ ), and non-CVD mortality (HR per SD [95\% CI]: 1.55 [1.30-1.84], $P<0.001)$. Conversely, uADMA is inversely associated with all-cause mortality (HR per SD [95\% CI]: 0.57 [0.47-0.69], $P<0.001$ ), CVD mortality (HR per SD [95\% CI]: 0.55 [0.40-0.74], $P<0.001$ ), and non-CVD mortality (HR per SD [95\% CI]: 0.59 [0.46-0.75], $P<0.001)$. Similar to uADMA, uSDMA is inversely associated with all-cause mortality (HR per SD [95\% CI]: 0.67 [0.55-0.82], P < 0.001), CVD mortality (HR per SD [95\% CI]: 0.63 [0.46-0.87], $P=0.01$ ), and
non-CVD mortality [HR per SD [95\% CI]: 0.70 [0.55-0.91], $P=0.01)$. The associations of pADMA with all-cause mortality and non-CVD mortality, and the associations of UADMA and USDMA with all-cause mortality and CVD mortality are independent of age, sex, eGFR, and potential confounders such as CVD risk factors and transplantationrelated factors. The HR of the associations of UADMA with outcomes remained relatively stable when adjusted for confounders (models 1-4), while the associations of pADMA with outcomes became considerably weaker.

In interaction analyses, we found a weak but significant interaction of pADMA with the presence of proteinuria $(P=0.02)$. The univariable association of pADMA with allcause mortality is stronger in RTR without proteinuria compared to those with proteinuria (events and HR per SD [95\% $\mathrm{CI}$ : 94 events and 1.54 [1.30-1.82] vs. 53 events and 1.22 
Table 2 Association of plasma ADMA and urinary excretions of ADMA and SDMA with all-cause mortality, cardiovascular mortality, and noncardiovascular mortality

\begin{tabular}{|c|c|c|c|c|c|c|}
\hline \multirow[b]{2}{*}{ All-cause } & \multicolumn{2}{|l|}{ pADMA } & \multicolumn{2}{|l|}{ uADMA } & \multicolumn{2}{|l|}{ uSDMA } \\
\hline & HR per SD $[95 \% \mathrm{CI}]$ & $P$ & HR per SD $[95 \% \mathrm{CI}]$ & $P$ & HR per SD $[95 \% \mathrm{CI}]$ & $P$ \\
\hline Crude & $1.45[1.26-1.67]$ & $<0.001$ & 0.57 [0.47-0.69] & $<0.001$ & $0.67[0.55-0.82]$ & $<0.001$ \\
\hline Model 1 & $1.29[1.09-1.52]$ & 0.004 & $0.67[0.49-0.91]$ & 0.011 & $0.76[0.62-0.93]$ & 0.01 \\
\hline Model 2 & $1.21[1.01-1.46]$ & 0.04 & $0.58[0.41-0.83]$ & 0.002 & $0.66[0.53-0.82]$ & $<0.001$ \\
\hline Model 3 & 1.24 [1.04-1.49] & 0.02 & $0.64[0.47-0.88]$ & 0.01 & $0.78[0.63-0.96]$ & 0.02 \\
\hline Model 4 & $1.27[1.07-1.50]$ & 0.01 & $0.66[0.48-0.90]$ & 0.01 & $0.79[0.65-0.97]$ & 0.02 \\
\hline Model 5 & $\mathrm{n} / \mathrm{a}$ & & $0.60[0.44-0.82]$ & 0.001 & $0.76[0.62-0.93]$ & 0.01 \\
\hline Model 5a & $1.36[1.15-1.62]$ & $<0.001$ & $\mathrm{n} / \mathrm{a}$ & & $0.82[0.65-1.03]$ & 0.09 \\
\hline Model 5b & $1.27[1.07-1.51]$ & 0.01 & $0.79[0.55-1.13]$ & 0.20 & $\mathrm{n} / \mathrm{a}$ & \\
\hline Model 6 & $1.33[1.12-1.58]$ & 0.001 & 0.69 [0.48-0.99] & 0.04 & $0.86[0.68-1.08]$ & 0.19 \\
\hline \multicolumn{7}{|c|}{ Cardiovascular } \\
\hline Crude & $1.30[1.03-1.65]$ & 0.03 & $0.55[0.40-0.74]$ & $<0.001$ & $0.63[0.46-0.87]$ & 0.01 \\
\hline Model 1 & $1.09[0.82-1.45]$ & 0.54 & $0.54[0.33-0.89]$ & 0.02 & $0.65[0.46-0.90]$ & 0.01 \\
\hline Model 2 & $0.97[0.72-1.32]$ & 0.86 & $0.53[0.30-0.92]$ & 0.03 & $0.59[0.42-0.83]$ & 0.002 \\
\hline Model 3 & $1.03[0.77-1.38]$ & 0.83 & $0.53[0.32-0.87]$ & 0.01 & $0.67[0.48-0.93]$ & 0.02 \\
\hline Model 4 & $1.08[0.81-1.43]$ & 0.60 & $0.54[0.32-0.90]$ & 0.02 & $0.67[0.48-0.93]$ & 0.02 \\
\hline Model 5 & $\mathrm{n} / \mathrm{a}$ & & $0.52[0.31-0.85]$ & 0.01 & $0.65[0.47-0.90]$ & 0.01 \\
\hline Model 5a & $1.18[0.89-1.56]$ & 0.25 & $\mathrm{n} / \mathrm{a}$ & & 0.71 [0.49-1.04] & 0.08 \\
\hline Model 5b & $1.10[0.83-1.46]$ & 0.51 & $0.74[0.42-1.31]$ & 0.30 & $\mathrm{n} / \mathrm{a}$ & \\
\hline Model 6 & $1.15[0.86-1.54]$ & 0.34 & $0.70[0.39-1.24]$ & 0.22 & $0.73[0.50-1.06]$ & 0.10 \\
\hline \multicolumn{7}{|c|}{ Non-cardiovascular } \\
\hline Crude & $1.55[1.30-1.84]$ & $<0.001$ & $0.59[0.46-0.75]$ & $<0.001$ & $0.70[0.55-0.91]$ & 0.01 \\
\hline Model 1 & $1.42[1.15-1.75]$ & 0.001 & $0.77[0.52-1.14]$ & 0.19 & 0.85 [0.66-1.09] & 0.19 \\
\hline Model 2 & $1.39[1.10-1.74]$ & 0.01 & $0.63[0.41-0.98]$ & 0.04 & $0.72[0.54-0.96]$ & 0.02 \\
\hline Model 3 & $1.39[1.10-1.76]$ & 0.01 & $0.73[0.48-1.10]$ & 0.13 & $0.87[0.67-1.15]$ & 0.33 \\
\hline Model 4 & $1.39[1.13-1.72]$ & 0.002 & $0.75[0.50-1.13]$ & 0.17 & $0.88[0.68-1.12]$ & 0.29 \\
\hline Model 5 & $\mathrm{n} / \mathrm{a}$ & & $0.67[0.45-0.99]$ & 0.04 & 0.85 [0.66-1.09] & 0.21 \\
\hline Model 5a & 1.49 [1.20-1.84] & $<0.001$ & $\mathrm{n} / \mathrm{a}$ & & 0.90 [0.67-1.19] & 0.45 \\
\hline Model 5b & $1.39[1.12-1.72]$ & 0.003 & $0.83[0.53-1.31]$ & 0.43 & $\mathrm{n} / \mathrm{a}$ & \\
\hline Model 6 & $1.45[1.17-1.81]$ & 0.001 & 0.69 [0.44-1.09] & 0.11 & $0.95[0.72-1.26]$ & 0.74 \\
\hline Model 1 & \multicolumn{6}{|c|}{ Crude + basic confounders (age, sex, BMI, eGFR ${ }^{\mathrm{a}}$, proteinuria) } \\
\hline Model 2 & \multicolumn{6}{|c|}{$\begin{array}{l}\text { Model } 1+\text { Framingham Risk Score factors (total cholesterol, HDL cholesterol, systolic blood pressure, antihypertensive } \\
\text { treatment, smoking (current, ex, or never), diabetes), medical history of coronary intervention, medical history of myocar- } \\
\text { dial infarction, and medical history of CVA and/or TIA }\end{array}$} \\
\hline Model 3 & \multicolumn{6}{|c|}{$\begin{array}{l}\text { Model } 1+\text { transplantation-related factors (donor type, total dialysis time, time from transplantation to baseline, cold } \\
\text { ischemia time, CNI usage, proliferation inhibitor usage, mTOR inhibitor usage, and number of transplantations up to } \\
\text { baseline) }\end{array}$} \\
\hline Model 4 & \multicolumn{6}{|c|}{ Model $1+$ primary disease } \\
\hline Model 5 & \multicolumn{6}{|l|}{ Model 1+pADMA } \\
\hline Model 5a & \multicolumn{6}{|l|}{ Model 1 + uADMA } \\
\hline Model 5b & \multicolumn{6}{|l|}{ Model 1+uSDMA } \\
\hline Model 6 & \multicolumn{6}{|c|}{ Model $1+$ uSDMA, uADMA, and pADMA combined } \\
\hline
\end{tabular}

pADMA plasma asymmetric dimethylarginine, $u A D M A$ urinary asymmetric dimethylarginine excretion $(24 \mathrm{~h})$, uSDMA urinary symmetric dimethylarginine excretion $(24 \mathrm{~h}), B M I$ body mass index, $e G F R$ estimated glomerular filtration rate, $H D L$ high-density lipoprotein, $C V A$ cerebrovascular accident, TIA transient ischemic attack, CNI calcineurin-inhibitor, mTOR mammalian target of rapamycin

${ }^{a}$ eGFR was calculated according to the Chronic Kidney Disease Epidemiology formula with serum creatinine and plasma cystatin C 
[0.96-1.56], respectively). Furthermore, there is a weak, but significant interaction of uSDMA with age for the association with non-CVD mortality $(P=0.01)$. The univariable association of uSDMA with non-CVD mortality is stronger for RTR with age above the median of 55 years compared to those with age equal to or below the median (events and HR per SD [95\% CI]: 70 events and $0.66[0.50-0.88]$ vs. 17 events and 1.14 [0.67-1.94], respectively).

\section{Model fit}

Although models with uADMA had comparable or slightly better fit for all-cause and CVD mortality, there is no significant difference in Harrell C statistic (Supplementary Table S1). For non-CVD mortality, pADMA has a slightly better fit, but the difference is not statistically significant. When both uADMA and pADMA are included in the same model, the associations of both pADMA and uADMA with outcomes not only remain significant, but become stronger (Table 2, model 5). Also, the association of uADMA with non-CVD mortality in the combined model remains independent of adjustment for potential confounders. There is a significant improvement in Harrell C statistics and -2 log likelihood for the combined pADMA and UADMA model vs. the single pADMA model for the unadjusted association with all-cause mortality (Supplementary Table S2: Harrell C: $P=0.03 ;-2 \log$ likelihood: $P<0.001)$. When adjusted for age, sex, BMI, eGFR, and proteinuria, the difference for Harrell C statistics is lost $(P=0.27)$, while it remains significant for the $-2 \log$ likelihood $(P=0.001)$. For the association with CVD mortality, there is no significant improvement of model fit according to the Harrell-C statistic when both pADMA and uADMA are included in the model. Yet there is an improvement for the model fit of pADMA when combined with uADMA according to the $-2 \log$ likelihood $(P<0.001$ and $P=0.01$ for unadjusted and adjusted models, respectively). For the association with non-CVD mortality, there is only a significant improvement in fit according to the Harrell-C statistic for the combined model in comparison with uADMA alone $(P=0.04)$. However, according to the $-2 \log$ likelihood, the model fit of both pADMA and uADMA for the association with non-CVD improves when they are combined.

\section{Associations with muscle and amino acid turnover markers}

In secondary analyses, we studied the associations of urinary creatinine and urea excretion with PADMA, UADMA, and uSDMA in a smaller subset of RTR who had mGFR data $(n=201)$. There are strongly positive and significant associations of urinary creatinine excretion and urea excretion with UADMA and uSDMA (Table 3), independent of age, sex, BMI, proteinuria, use of mammalian target of rapamycin (mTOR) inhibitors and mGFR (creatinine: st. $\beta: 0.27$, $P<0.001$; urea: st. $\beta$ : $0.30, P<0.001)$. Urinary creatinine is also (negatively) associated with pADMA, while urinary urea excretion is not associated with pADMA (Table 3; st. $\beta:-0.20, P=0.03$ and st. $\beta:-0.02, P=0.81$, respectively).

In further analyses, we adjusted the associations of pADMA, uADMA, and uSDMA with long-term outcomes for urinary creatinine excretion and urinary urea excretion (Table 4). The associations of pADMA, uADMA, and uSDMA with all-cause mortality and CVD mortality are highly dependent upon urinary creatinine excretion: the increased risk of pADMA with all-cause and non-CVD mortality is reduced by a third, while the associations of UADMA and USDMA with all-cause and CVD mortality are lost. Similar to urinary creatinine excretion, but to a lesser extent, the association of pADMA with all-cause mortality is also dependent upon 24-h urinary urea excretion.

There are significant interactions between urinary creatinine and urea excretion with UADMA for the association with CVD mortality (interaction coefficients with uADMA: urinary creatinine: $0.07, P=0.01$; urinary urea: 0.07 , $P=0.01)$. There is no significant interaction coefficient of uADMA, USDMA or pADMA with urinary creatinine or urea excretion for the associations with all-cause and nonCVD mortality.

\section{Discussion}

To our knowledge, we have shown for the first time an inverse association of UADMA and of USDMA with allcause and CVD mortality in a large, prospective cohort study of RTR. Unadjusted, there is a risk reduction of $43 \%, 45 \%$, and $41 \%$ per standard deviation increment of uADMA for all-cause, CVD, and non-CVD mortality, respectively. The associations with all-cause and CVD mortality are independent of adjustment for potential confounders. Results for analyses with uSDMA were comparable to those for uADMA. pADMA was associated with an increase in all-cause and non-CVD mortality, but not with CVD mortality. In secondary analyses, we have found that pADMA, uADMA, and uSDMA are strongly and independently associated with urinary creatinine excretion. Additionally, uADMA and uSDMA are strongly associated with urinary urea excretion. The associations of UADMA and USDMA with all-cause, CVD, and non-CVD mortality are highly dependent upon urinary creatinine excretion and urinary urea excretion.

The mean pADMA concentration in the RTR population of our study is comparable to that of the general public (Atzler et al. 2014). The association of pADMA with CVD and renal outcomes has been demonstrated in the literature in CKD patients and the general population (Zoccali et al. 
Table 3 Associations of urinary creatinine and urea excretions with plasma ADMA and urinary excretions of ADMA and SDMA in a subpopulation of $\operatorname{RTR}(n=201)$

\begin{tabular}{|c|c|c|c|c|}
\hline \multirow{3}{*}{$\begin{array}{l}\text { Dependent } \\
\text { Predictor }\end{array}$} & \multicolumn{4}{|c|}{ pADMA } \\
\hline & \multicolumn{2}{|c|}{ Urinary creatinine excretion } & \multicolumn{2}{|c|}{ Urinary urea excretion } \\
\hline & St. $\beta$ & $P$ & St. $\beta$ & $P$ \\
\hline Crude & -0.21 & 0.003 & -0.10 & 0.14 \\
\hline Model 1 & -0.30 & 0.001 & -0.12 & 0.11 \\
\hline Model 2 & -0.20 & 0.03 & -0.02 & 0.81 \\
\hline Dependent & \multicolumn{4}{|c|}{ uADMA } \\
\hline \multirow[t]{2}{*}{ Predictor } & \multicolumn{2}{|c|}{ Urinary creatinine excretion } & \multicolumn{2}{|c|}{ Urinary urea excretion } \\
\hline & St. $\beta$ & $P$ & St. $\beta$ & $P$ \\
\hline Crude & 0.49 & $<0.001$ & 0.56 & $<0.001$ \\
\hline Model 1 & 0.55 & $<0.001$ & 0.52 & $<0.001$ \\
\hline Model 2 & 0.27 & $<0.001$ & 0.30 & $<0.001$ \\
\hline Dependent & uSDM & & & \\
\hline \multirow[t]{2}{*}{ Predictor } & \multicolumn{2}{|c|}{ Urinary creatinine excretion } & \multicolumn{2}{|c|}{ Urinary urea excretion } \\
\hline & St. $\beta$ & $P$ & St. $\beta$ & $P$ \\
\hline Crude & 0.52 & $<0.001$ & 0.47 & $<0.001$ \\
\hline Model 1 & 0.36 & $<0.001$ & 0.41 & $<0.001$ \\
\hline Model 2 & 0.29 & $<0.001$ & 0.34 & $<0.001$ \\
\hline Model 1 & \multicolumn{4}{|c|}{ Crude + age, sex, BMI, proteinuria, and use of mTOR inhibitors } \\
\hline Model 2 & \multicolumn{4}{|c|}{ Model $1+$ mGFR } \\
\hline
\end{tabular}

pADMA plasma asymmetric dimethylarginine, uADMA urinary asymmetric dimethylarginine excretion $(24 \mathrm{~h}), u S D M A$ urinary symmetric dimethylarginine excretion $(24 \mathrm{~h}), m T O R$ mammalian target of rapamycin, $m G F R$ measured GFR (by ${ }^{125}$ I-iothalamate)

Table 4 Effect of urinary creatinine and urea excretion on the associations of plasma ADMA and urinary excretions of ADMA and SDMA with mortality, cardiovascular mortality, and noncardiovascular mortality

\begin{tabular}{|c|c|c|c|c|c|c|}
\hline & \multicolumn{2}{|l|}{ pADMA } & \multicolumn{2}{|l|}{ uADMA } & \multicolumn{2}{|l|}{ uSDMA } \\
\hline & HR per SD $[95 \% \mathrm{CI}]$ & $P$ & HR per SD $[95 \% \mathrm{CI}]$ & $P$ & HR per SD $[95 \% \mathrm{CI}]$ & $P$ \\
\hline \multicolumn{7}{|l|}{ All-cause } \\
\hline Base & $1.30[1.10-1.55]$ & 0.002 & $0.68[0.50-0.92]$ & 0.01 & $0.77[0.62-0.94]$ & 0.01 \\
\hline Model 1 & $1.19[1.01-1.42]$ & 0.04 & $1.14[0.81-1.60]$ & 0.46 & $0.98[0.80-1.21]$ & 0.87 \\
\hline Model 2 & 1.26 [1.06-1.49] & 0.01 & $0.97[0.69-1.36]$ & 0.87 & $0.94[0.75-1.17]$ & 0.55 \\
\hline \multicolumn{7}{|c|}{ Cardiovascular } \\
\hline Base & $1.10[0.83-1.46]$ & 0.52 & $0.54[0.33-0.89]$ & 0.02 & $0.65[0.47-0.90]$ & 0.01 \\
\hline Model 1 & $1.00[0.75-1.33]$ & 0.99 & $0.87[0.50-1.51]$ & 0.62 & $0.81[0.57-1.16]$ & 0.25 \\
\hline Model 2 & $1.06[0.80-1.42]$ & 0.69 & $0.69[0.39-1.20]$ & 0.19 & $0.73[0.50-1.05]$ & 0.09 \\
\hline \multicolumn{7}{|c|}{ Non-cardiovascular } \\
\hline Base & $1.45[1.17-1.79]$ & 0.001 & $0.78[0.53-1.17]$ & 0.23 & $0.86[0.66-1.11]$ & 0.23 \\
\hline Model 1 & $1.33[1.07-1.65]$ & 0.01 & $1.37[0.89-2.12]$ & 0.15 & $1.10[0.87-1.41]$ & 0.43 \\
\hline Model 2 & $1.39[1.12-1.72]$ & 0.003 & $1.22[0.79-1.87]$ & 0.37 & $1.09[0.84-1.42]$ & 0.51 \\
\hline Base & \multicolumn{6}{|c|}{ Crude + age, sex, BMI, eGFR ${ }^{\mathrm{a}}$, proteinuria, and use of mTOR inhibitors } \\
\hline Model 1 & \multicolumn{6}{|c|}{ Base $+24 \mathrm{~h}$ urinary creatinine excretion } \\
\hline Model 2 & \multicolumn{6}{|c|}{ Base $+24 \mathrm{~h}$ urinary urea excretion } \\
\hline
\end{tabular}

PADMA plasma asymmetric dimethylarginine, $u A D M A$ urinary asymmetric dimethylarginine excretion (24 h), uSDMA urinary symmetric dimethylarginine excretion $(24 \mathrm{~h}), B M I$ body mass index, eGFR estimated glomerular filtration rate, mTOR mammalian target of rapamycin

${ }^{a}$ eGFR was calculated according to the Chronic Kidney Disease Epidemiology formula with serum creatinine and plasma cystatin $\mathrm{C}$ 
2001; Achan et al. 2003; Fliser et al. 2005), but we are the first to present the associations between uADMA and longterm outcomes. For the RTR population, we revealed earlier in the same cohort the association between higher pADMA and all-cause mortality with a shorter follow-up (median 3.1 vs. 5.4 years) (Frenay et al. 2015). In the present study, we have demonstrated that UADMA is a more consistent predictor of mortality compared to pADMA, with hazard ratio remaining relatively stable when adjusted for potential confounders, while the hazard ratio for pADMA fluctuates more depending on the adjustment. Furthermore, we have shown that pADMA and uADMA strengthen each other's association with long-term outcomes when put together in a statistical model. This may be explained by an inverse collinearity, where pADMA is dependent upon the ability to be renally excreted; pADMA increases as uADMA decreases. Then, one may suggest that we may be looking at the effect of renal ADMA clearance and thereby renal function rather than the individual effects of pADMA and uADMA in the presented models. The importance of kidney function in the homeostasis of circulatory ADMA has been emphasized repeatedly in the literature, as several studies have observed that pADMA increases with worsening renal function. However, the reality is far more complex since only a fraction of about $17 \%$ of exogenous circulating ADMA (intravenous injection of $3 \mathrm{mg} / \mathrm{kg}$ ) is excreted unchanged in the urine (Achan et al. 2003). The remainder is largely metabolized by dimethylarginine dimethylaminohydrolase (DDAH) to citrulline and dimethylamine (DMA), with the latter being excreted in the urine (Achan et al. 2003). A small portion of ADMA is transaminated by the mitochondrial aminotransferase alanine-glyoxylate aminotransferase 2 (AGXT2) to $\alpha$-keto- $\delta$-( $N^{\mathrm{G}}, N^{\mathrm{G}}$-dimethylguanidino) valeric acid (Rodionov et al. 2010; Morris 2016). Diminished activity of DDAH and AGXT2 has been suggested as the main reason of pADMA accumulation in the blood rather than lower renal clearance (Matsuguma et al. 2006; Kayacelebi et al. 2015). We have recently published a study in kidney donors that demonstrated an acute renal function impairment resulting from kidney donation drastically reduced the urinary excretion of ADMA, but had a minimal effect on plasma ADMA (Said et al. 2019).

Being an NOS inhibitor, pADMA has been traditionally associated with cardiovascular outcomes (Kielstein and Zoccali 2005). We found that not pADMA, but uADMA is strongly associated with CVD mortality in RTR. Similarly, Böger et al. (2009) found no significant association of pADMA with cardiovascular death in 3320 normal subjects from the Framingham Offspring Study (Böger et al. 2009). Wolf et al. (2012) found that a high ADMA/creatinine concentration in spot urine samples was strongly protective against cardiac death in a cardiovascular risk population (Wolf et al. 2012). The findings of Böger et al. and Wolf et al. corroborate our findings. Furthermore, we have demonstrated that the associations revealed for uSDMA closely resemble those for uADMA. uSDMA is known to be almost exclusively eliminated by renal excretion (Nijveldt et al. 2002). While only ADMA can be metabolized by DDAH, both ADMA and SDMA can be metabolized by AGXT2. Recently, AGXT2 activity has been found to be associated with worse clinical outcomes in CKD patients (MartensLobenhoffer et al. 2018). It had been suggested that higher AGXT2 activity may be a response to elevated systemic ADMA and SDMA levels, possibly due to decreased renal excretion. In the current study, given that the associations of USDMA and UADMA are closely alike and significant despite adjustment for renal function, it is possible that variation in AGXT2 activity is the driving force underlying the found associations. It would suggest that high AGXT2 (and thereby low UADMA and low USDMA) would indeed be associated with worse outcomes. The underlying pathophysiology remains speculative, however. So far, uSDMA has not been found to have significant direct NO-inhibitory properties. However, it has been suggested that uSDMA can compete with L-arginine transport, having an indirect inhibitory property (Bode-Böger et al. 2006).

The production of ADMA and SDMA depends on two subsequent steps of post-translational arginine methylation mediated by a family of methyltransferases [protein arginine methyltransferases (PRMTs)]. Of these, nine PRMTs have been implicated in catalyzing the first step of arginine residue methylation to monomethylated arginine (MMA) in mammalian cells (Blanc and Richard 2017). Based on the ability to catalyze a subsequent methylation step, the PRMTs can be subdivided in three types. Type 1 PRMTs are represented by six members of the methyltransferase family (PRMT1-4, 6, 8) and generate ADMA, while type 2 PRMTs are represented by two members of the methyltransferase family (PRMT5, 9) and generate SDMA (Tang et al. 1998; Branscombe et al. 2001; Frankel et al. 2002; Lee et al. 2005; Zurita-Lopez et al. 2012; Dhar et al. 2013; Yang et al. 2015; Cura et al. 2017; Shishkova et al. 2017). Type 3 PRMTs are represented by a single member of the methyltransferase family (PRMT7) and do not catalyze a subsequent methylation step (Zurita-Lopez et al. 2012)), which causes them to only produce MMA and thus far only histones are known substrates for this enzyme (Feng et al. 2013; Blanc and Richard 2017). In general, virtually all types of cell are capable of producing ADMA, presumably because ADMA originates from the turnover of argininemethylated proteins, which are apparently present in all cell types (Teerlink 2005). In this respect, we attempted to study the associations between ADMA, and urinary creatinine and urea excretion. In biochemically stable conditions, urinary creatinine excretion measured in 24-h urine is accepted as a reflection of total body muscle mass (Wyss 
and Kaddurah-Daouk 2000). Creatinine is produced from the likely irreversible, non-enzymatic dehydration of creatine, which is necessary for the phosphocreatine buffer of the cellular energy provision (Wyss and Kaddurah-Daouk 2000). Muscular tissue provides by far most of body creatine from the turnover of muscle proteins (Hunter 1922; Wyss and Kaddurah-Daouk 2000); a high 24-h urinary creatinine excretion in stable conditions implies a large muscle protein turnover resulting from larger muscle mass (Heymsfield et al. 1983). Therefore, urinary creatinine excretion may reflect the production of ADMA from muscle protein turnover too, as represented by the positive association of urinary creatinine excretion with uADMA. Alternatively, it may be that muscle mass maintenance is dependent upon ADMA homeostasis, although this has scarcely been studied. The negative and weaker association of pADMA with urinary creatinine excretion may be explained due to this mechanism. Muscle synthesis is mediated by the activation of the protein kinase B-mTOR (Schiaffino et al. 2013). One of the pathways that activate mTOR is by triggering neuronal NOS (nNOS) (Ito et al. 2013). ADMA is a universal NOS inhibitor and a strong inhibitor of nNOS activity (Kielstein et al. 2007), which may then negatively affect muscle growth. Ito et al. (2013) performed a study in nNOS-null mice and showed that nNOS-null mice not only had less muscle mass increase in response to load stimulation compared to wildtype mice, but that the administration of $N^{\mathrm{G}}$-nitro-L-arginine methyl ester (L-NAME), an exogenous inhibitor of all NOS isoforms, prevented an increase in muscle mass in wild-type mice (Ito et al. 2013). Wild-type mice that were administered the enantiomer D-NAME, which does not inhibit NOS activity, did increase in muscle mass. Frandsen et al. (2001) have studied L-NAME infusion in six healthy young men and found a $67 \%$ lower NOS activity in skeletal muscle biopsies after infusion compared to control biopsies (Frandsen et al. 2001). However, whether an acute inhibition of NOS activity will have a noticeable acute effect on muscle mass is questionable. To our knowledge, no studies have been performed in human participants that have studied the effects of NOS inhibition on muscle mass. We found a very strong positive association of urinary creatinine excretion with uADMA and a negative association with pADMA, independent of age, sex, BMI, proteinuria, mTOR inhibitors, and mGFR in a smaller subset of our study population. It is likely that both mechanisms of the interaction of muscle mass and ADMA play a role, possibly leaning more to the side of muscle mass being a source of ADMA since the positive association of urinary creatinine excretion with uADMA is much stronger than the negative association with pADMA. Last, the negative association between pADMA and urinary creatinine excretion may be due to residual confounding and promotes the need for further research into the interplay between pADMA and creatinine excretion.
In the current study, urinary urea excretion was strongly associated with uADMA, but not with pADMA. That urea excretion is more likely a nutritional marker than an amino acid turnover marker may partly explain this. Urea is produced from ammonia in amino acid metabolism and 24-h urinary urea excretion may be viewed as a marker of not only amino acid turnover, but also average dietary protein intake and thereby a reflection of an independent dietary ADMA source itself (Weiner et al. 2015). pADMA is relatively independent of nutritional intake, as demonstrated by the study of Schneider et al. (2015), who found that daily oral arginine supplementation $(9.96 \mathrm{~g} / 24 \mathrm{~h})$ had little effect on pADMA concentration after 3 months in patients with peripheral occlusive artery disease and after 6 months in patients with coronary artery disease compared to placebo (Schneider et al. 2015). This may be due to increased compensatory ADMA metabolism and/or due to increased elimination via the renal route. Therefore, uADMA may be more reflective of dietary ADMA intake than pADMA. Furthermore, it is important to remember that dietary protein, reflected by urinary urea excretion, is also a substrate for muscle protein synthesis, and urea excretion has been strongly correlated to muscle mass before (Said et al. 2015). Therefore, urea excretion may be similarly reflective of ADMA production from muscle protein turnover. When the associations of uADMA and pADMA with mortality are adjusted for creatinine and urea excretion, the associations become considerably weaker. We hypothesize, therefore, that muscle turnover and protein intake play a significant role in the production, homeostasis, and pathogenesis of ADMA.

Strengths of the present study are the large study population and the long follow-up. The prospective study design and the large variety of measured variables enabled us to adjust for many confounding risk factors at baseline. A limitation of this study is that the exact origin of pADMA or UADMA is unknown. It may be possible that urinary ADMA excretion reflects more renal ADMA production rather than systemic ADMA production. The strong, independent associations with urinary creatinine and urea excretion, and parameters reflecting systemic ADMA sources are in favor of the theory that pADMA and UADMA reflect systemic homeostasis, although further studies are needed to independently assess specific renal ADMA production and provide clarity. Nevertheless, the results of this study are relevant as they demonstrate that UADMA is not only a strongly significant, but also a more consistent predictor of long-term outcomes than pADMA. It would have been interesting to study how plasma SDMA would compare to pADMA. A potential role for arginine methylation in cardiovascular disease largely remained indirect via the inhibition of endogenous NO synthase in endothelial cells by MMA and ADMA (Stühlinger et al. 2001; Peng and Wong 2017), but recent 
evidence also suggests a more direct role. It has, for example, been found that arginine methylation mediated by PRMT3 and PRMT5 regulates the expression of the cardiac voltage-gated sodium channel $\mathrm{Na}_{\mathrm{v}} 1.5$, which may be implicated in the development of heart failure (BeltranAlvarez et al. 2013, 2014). Inclusion of plasma SDMA data complemented by urinary SDMA excretion data, in addition to plasma ADMA data complemented by urinary ADMA excretion data, might also have given information on the balance between activities of type 1 and type 2 PRMTs. However, for the current study it was not possible to measure SDMA in plasma, since the applied method of GC-MS/MS does not allow for accurate measurement of SDMA in plasma. Although GC-MS/MS provides a reliable and accurate method to measure plasma and urinary ADMA (Tsikas et al. 2003), and has recently been demonstrated to be also suitable for measuring urinary SDMA (Bollenbach et al. 2018), it is not suitable for measurement of plasma SDMA (personal communication).

A further limitation is that our study was observational in nature, which makes that the associations unveiled do not necessarily imply causation.

In conclusion, high uADMA and high uSDMA are associated with lower risk of all-cause mortality and less cardiovascular mortality in RTR. Higher muscle mass and protein intake are associated with lower pADMA levels and higher UADMA and USDMA excretion. The association of pADMA, uADMA, and uSDMA with long-term outcomes in RTR is also highly dependent on markers of muscle mass and protein intake. Our results suggest that an increase in muscle mass and amino acid turnover is associated with a beneficial increase in UADMA without an increase in pADMA, even when adjusted for renal function. The exact mechanism of these associations needs further clarification in future studies. However, our data present opportunities to influence the negative effects of ADMA through potentially modifiable factors, namely muscle mass and dietary protein intake. An intervention involving the modification of muscle mass and dietary protein intake, and their influence on ADMA and SDMA homeostasis is warranted. Clinical implications of this study may be to stimulate muscle mass growth, possibly by increased protein intake and physical activity, to augment ADMA and SDMA excretion and to reduce risk of premature mortality.

\section{Compliance with ethical standards}

Conflict of interest The authors declare they have no conflict of interest.

Ethical approval All procedures performed in this study were in accordance with the ethical standards of the institutional and national research committee and with the 1964 Helsinki declaration and its later amendments or comparable ethical standards. This article does not contain any studies with animals performed by any of the authors.

Informed consent We obtained informed consent from all individual participants included in the study.

Open Access This article is distributed under the terms of the Creative Commons Attribution 4.0 International License (http://creativeco mmons.org/licenses/by/4.0/), which permits unrestricted use, distribution, and reproduction in any medium, provided you give appropriate credit to the original author(s) and the source, provide a link to the Creative Commons license, and indicate if changes were made.

\section{References}

Achan V, Broadhead M, Malaki M et al (2003) Asymmetric dimethylarginine causes hypertension and cardiac dysfunction in humans and is actively metabolized by dimethylarginine dimethylaminohydrolase. Arterioscler Thromb Vasc Biol 23:1455-1459. https:// doi.org/10.1161/01.ATV.0000081742.92006.59

Apperloo AJ, de Zeeuw D, Donker AJ, de Jong PE (1996) Precision of glomerular filtration rate determinations for long-term slope calculations is improved by simultaneous infusion of ${ }^{125} \mathrm{I}$-iothalamate and 131I-hippuran. J Am Soc Nephrol 7:567-572

Atzler D, Schwedhelm E, Nauck M et al (2014) Serum reference intervals of homoarginine, ADMA, and SDMA in the Study of Health in Pomerania. Clin Chem Lab Med 52:1835-1842. https://doi. org/10.1515/cclm-2014-0314

Beltran-Alvarez P, Espejo A, Schmauder R et al (2013) Protein arginine methyl transferases-3 and -5 increase cell surface expression of cardiac sodium channel. FEBS Lett 587:3159-3165. https://doi. org/10.1016/j.febslet.2013.07.043

Beltran-Alvarez P, Tarradas A, Chiva C et al (2014) Identification of N-terminal protein acetylation and arginine methylation of the voltage-gated sodium channel in end-stage heart failure human heart. Curr Ther Res Clin Exp 76:126-129. https://doi. org/10.1016/j.yjmcc.2014.08.014

Blanc RS, Richard S (2017) Arginine methylation: the coming of age. Mol Cell 65:8-24. https://doi.org/10.1016/j.molcel.2016.11.003

Bode-Böger SM, Scalera F, Kielstein JT et al (2006) Symmetrical dimethylarginine: a new combined parameter for renal function and extent of coronary artery disease. J Am Soc Nephrol 17:11281134. https://doi.org/10.1681/asn.2005101119

Sullivan LM, Schwedhelm E et al (2009) Plasma asymmetric dimethylarginine and incidence of cardiovascular disease and death in the community. Circulation 119:1592-1600. https://doi.org/10.1161/ circulationaha.108.838268

Bollenbach A, Hanff E, Beckmann B et al (2018) GC-MS quantification of urinary symmetric dimethylarginine (SDMA), a wholebody symmetric L-arginine methylation index. Anal Biochem 556:40-44. https://doi.org/10.1016/j.ab.2018.06.021

Branscombe TL, Frankel A, Lee JH et al (2001) PRMT5 (Janus kinasebinding protein 1) catalyzes the formation of symmetric dimethylarginine residues in proteins. J Biol Chem 276:32971-32976. https://doi.org/10.1074/jbc.M105412200

Cura V, Marechal N, Troffer-Charlier N et al (2017) Structural studies of protein arginine methyltransferase 2 reveal its interactions with potential substrates and inhibitors. FEBS J 284:77-96. https://doi. org/10.1111/febs. 13953

Dhar S, Vemulapalli V, Patananan AN et al (2013) Loss of the major type I arginine methyltransferase PRMT1 causes substrate 
scavenging by other PRMTs. Sci Rep 3:1311. https://doi. org/10.1038/srep01311

Du Bois D, Du Bois EF (1916) A formula to estimate the approximate surface area if height and weight be known 1916. Nutrition 5:303311. https://doi.org/10.1001/archinte.1916.00080130010002

Feng Y, Maity R, Whitelegge JP et al (2013) Mammalian protein arginine methyltransferase 7 (PRMT7) specifically targets RXR sites in lysine- and arginine-rich regions. J Biol Chem 288:37010 37025. https://doi.org/10.1074/jbc.m113.525345

Fliser D, Kronenberg F, Kielstein JT et al (2005) Asymmetric dimethylarginine and progression of chronic kidney disease: the mild to moderate kidney disease study. J Am Soc Nephrol 16:2456-2461. https://doi.org/10.1681/asn.2005020179

Frandsenn U, Bangsbo J, Sander M et al (2001) Exercise-induced hyperaemia and leg oxygen uptake are not altered during effective inhibition of nitric oxide synthase with $\mathrm{N}(\mathrm{G})$-nitro-L-arginine methyl ester in humans. J Physiol 531:257-264. https://doi. org/10.1111/j.1469-7793.2001.0257j.x

Frankel A, Yadav N, Lee J et al (2002) The novel human protein arginine $N$-methyltransferase PRMT6 is a nuclear enzyme displaying unique substrate specificity. J Biol Chem 277:3537-3543. https://doi.org/10.1074/jbc.M108786200

Frenay AR, van den Berg E, de Borst MH et al (2015) Plasma ADMA associates with all-cause mortality in renal transplant recipients. Amino Acids 47:1941-1949. https://doi.org/10.1007/ s00726-015-2023-0

Heymsfield SB, Arteaga C, McManus C et al (1983) Measurement of muscle mass in humans: validity of the 24-h urinary creatinine method. Am J Clin Nutr 37:478-494. https://doi.org/10.1093/ ajen/37.3.478

Hunter A (1922) The physiology of creatine and creatinine. Physiol Rev 2:586-626. https://doi.org/10.1152/physrev.1922.2.4.586

Ito N, Ruegg UT, Kudo A et al (2013) Activation of calcium signaling through Trpv1 by nNOS and peroxynitrite as a key trigger of skeletal muscle hypertrophy. Nat Med 19:101-106. https:// doi.org/10.1038/nm.3019

Jacobi J, Tsao PS (2008) Asymmetrical dimethylarginine in renal disease: limits of variation or variation limits? A systematic review. Am J Nephrol 28:224-237. https://doi.org/10.1159/000110092

Kayacelebi AA, Langen J, Weigt-Usinger K et al (2015) Biosynthesis of homoarginine (hArg) and asymmetric dimethylarginine (ADMA) from acutely and chronically administered free L-arginine in humans. Amino Acids 47:1893-1908. https://doi. org/10.1007/s00726-015-2012-3

Kielstein JT, Zoccali C (2005) Asymmetric dimethylarginine: a cardiovascular risk factor and a uremic toxin coming of age? Am J Kidney Dis 46:186-202. https://doi.org/10.1053/j.ajkd.2005.05.009

Kielstein A, Tsikas D, Galloway GP, Mendelson JE (2007) Asymmetric dimethylarginine (ADMA) - a modulator of nociception in opiate tolerance and addiction? Nitric Oxide. https://doi.org/10.1016/j. niox.2007.05.005

Lee J, Sayegh J, Daniel J et al (2005) PRMT8, a new membrane-bound tissue-specific member of the protein arginine methyltransferase family. J Biol Chem 280:32890-32896. https://doi.org/10.1074/ jbc.M506944200

Maroni BJ, Steinman TI, Mitch WE (1985) A method for estimating nitrogen intake of patients with chronic renal failure. Kidney Int 27:58-65. https://doi.org/10.1038/ki.1985.10

Martens-Lobenhoffer J, Emrich IE, Zawada AM et al (2018) L-Homoarginine and its AGXT2-metabolite GOCA in chronic kidney disease as markers for clinical status and prognosis. Amino Acids 50:1347-1356. https://doi.org/10.1007/s00726-018-2610-y

Matsuguma K, Ueda S, Yamagishi S-I et al (2006) Molecular mechanism for elevation of asymmetric dimethylarginine and its role for hypertension in chronic kidney disease. J Am Soc Nephrol 17:2176-2183. https://doi.org/10.1681/asn.2005121379
Minović I, van der Veen A, van Faassen M et al (2017) Functional vitamin B-6 status and long-term mortality in renal transplant recipients. Am J Clin Nutr 106:1366-1374. https://doi.org/10.3945/ ajen.117.164012

Morris SM (2016) Arginine metabolism revisited. J Nutr 146:2579S-2586S. https://doi.org/10.3945/jn.115.226621

National Center for Health Statistics (NCHS) and the Centers for Medicare and Medicaid Services (CMS) (2011) The International Classification of Diseases, 9th Revision, Clinical Modification: ICD. 9. CM. Ann Arbor, Michigan, USA

Nijveldt RJ, Van Leeuwen PAM, Van Guldener C et al (2002) Net renal extraction of asymmetrical (ADMA) and symmetrical (SDMA) dimethylarginine in fasting humans. Nephrol Dial Transplant 17:1999-2002

Peng C, Wong CC (2017) The story of protein arginine methylation: characterization, regulation, and function. Expert Rev Proteom 14:157-170. https://doi.org/10.1080/14789450.2017.1275573

Rodionov RN, Murry DJ, Vaulman SF et al (2010) Human alanineglyoxylate aminotransferase 2 lowers asymmetric dimethylarginine and protects from inhibition of nitric oxide production. J Biol Chem 285:5385-5391. https://doi.org/10.1074/jbc.M109.091280

Said MY, Deetman PE, de Vries APJ et al (2015) Causal path analyses of the association of protein intake with risk of mortality and graft failure in renal transplant recipients. Clin Transplant 29:447-457. https://doi.org/10.1111/ctr.12536

Said MY, Douwes RM, van Londen M et al (2019) Effect of renal function on homeostasis of asymmetric dimethylarginine (ADMA): studies in donors and recipients of renal transplants. Amino Acids 1:3. https://doi.org/10.1007/s00726-018-02693-Z

Saran R, Robinson B, Abbott K et al (2018) US renal data system 2017 annual data report: epidemiology of kidney disease in the United States. Am J Kidney Dis 71:(suppl 1):Svii, S1-S672

Schiaffino S, Dyar KA, Ciciliot S et al (2013) Mechanisms regulating skeletal muscle growth and atrophy. FEBS J 280:4294-4314. https ://doi.org/10.1111/febs.12253

Schneider JY, Rothmann S, Schröder F et al (2015) Effects of chronic oral $\mathrm{L}$-arginine administration on the $\mathrm{L}$-arginine/NO pathway in patients with peripheral arterial occlusive disease or coronary artery disease: L-Arginine prevents renal loss of nitrite, the major NO reservoir. Amino Acids 47:1961-1974. https://doi. org/10.1007/s00726-015-2031-0

Shishkova E, Zeng H, Liu F et al (2017) Global mapping of CARM1 substrates defines enzyme specificity and substrate recognition. Nat Commun 8:15571. https://doi.org/10.1038/ncomms 15571

Stühlinger MC, Tsao PS, Her JH et al (2001) Homocysteine impairs the nitric oxide synthase pathway: role of asymmetric dimethylarginine. Circulation 104:2569-2575. https://doi.org/10.1161/ hc4601.098514

Tang J, Gary JD, Clarke S, Herschman HR (1998) PRMT 3, a type I protein arginine $N$-methyltransferase that differs from PRMT1 in its oligomerization, subcellular localization, substrate specificity, and regulation. J Biol Chem 273:16935-16945. https://doi. org/10.1074/jbc.273.27.16935

Teerlink T (2005) ADMA metabolism and clearance. Vasc Med 10(Suppl 1):S73-S81. https://doi.org/10.1191/1358863x05vm597 oa

Tsikas D, Schubert B, Gutzki F-M et al (2003) Quantitative determination of circulating and urinary asymmetric dimethylarginine (ADMA) in humans by gas chromatography-tandem mass spectrometry as methyl ester tri( $N$-pentafluoropropionyl) derivative. J Chromatogr B Analyt Technol Biomed Life Sci 798:87-99. https ://doi.org/10.1016/j.jchromb.2003.09.001

van den Berg E, Engberink MF, Brink EJ et al (2012) Dietary acid load and metabolic acidosis in renal transplant recipients. Clin J Am Soc Nephrol 7:1811-1818. https://doi.org/10.2215/CJN.04590512 
van den Berg E, Engberink MF, Brink EJ et al (2013) Dietary protein, blood pressure and renal function in renal transplant recipients. Br J Nutr 109:1463-1470. https://doi.org/10.1017/S000711451 2003455

van den Berg E, Pasch A, Westendorp WH et al (2014) Urinary sulfur metabolites associate with a favorable cardiovascular risk profile and survival benefit in renal transplant recipients. J Am Soc Nephrol 25:1303-1312. https://doi.org/10.1681/ASN.2013050497

Weiner ID, Mitch WE, Sands JM (2015) Urea and ammonia metabolism and the control of renal nitrogen excretion. Clin J Am Soc Nephrol 10:1444-1458. https://doi.org/10.2215/CJN.10311013

Wolf C, Lorenzen JM, Stein S et al (2012) Urinary asymmetric dimethylarginine (ADMA) is a predictor of mortality risk in patients with coronary artery disease. Int J Cardiol 156:289-294. https://doi. org/10.1016/j.ijcard.2010.11.003

Wyss M, Kaddurah-Daouk R (2000) Creatine and creatinine metabolism. Physiol Rev 80:1107-1213. https://doi.org/10.1152/physr ev.2000.80.3.1107
Yang Y, Hadjikyriacou A, Xia Z et al (2015) PRMT9 is a type II methyltransferase that methylates the splicing factor SAP145. Nat Commun 6:6428. https://doi.org/10.1038/ncomms 7428

Zoccali C, Bode-Böger S, Mallamaci F et al (2001) Plasma concentration of asymmetrical dimethylarginine and mortality in patients with end-stage renal disease: a prospective study. Lancet 358:2113-2117. https://doi.org/10.1016/S0140-6736(01)07217 -8 (London, England)

Zoccali C, Benedetto FA, Maas R et al (2002) Asymmetric dimethylarginine, $\mathrm{C}$-reactive protein, and carotid intima-media thickness in end-stage renal disease. J Am Soc Nephrol 13:490-496

Zurita-Lopez CI, Sandberg T, Kelly R, Clarke SG (2012) Human protein arginine methyltransferase 7 (PRMT7) is a type III enzyme forming $\omega$-NG-monomethylated arginine residues. J Biol Chem 287:7859-7870. https://doi.org/10.1074/jbc.M111.336271

Publisher's Note Springer Nature remains neutral with regard to jurisdictional claims in published maps and institutional affiliations.

\section{Affiliations}

\section{Yusof Said ${ }^{1}$ - A. Bollenbach ${ }^{2}$ Isidor Minović ${ }^{3} \cdot$ Marco van Londen $^{1}$ • Anne-Roos Frenay ${ }^{4} \cdot$ Martin H. de Borst ${ }^{1,6}$. Else van den Berg ${ }^{1} \cdot$ A. Arinc Kayacelebi ${ }^{2} \cdot$ Dimitrios Tsikas $^{2} \cdot$ Harry van Goor ${ }^{5,6} \cdot$ Gerjan Navis $^{1,6} \cdot$ Stephan J. \\ L. Bakker ${ }^{1,6}$}

1 Department of Internal Medicine, Division of Nephrology, University Medical Center Groningen, Sector A, University of Groningen, PO Box 30.001, Hanzeplein 1, 9700 RB Groningen, The Netherlands

2 Institute of Toxicology, Core Unit Proteomics, Hannover Medical School, Hannover, Germany

3 Department of Laboratory Medicine, University Medical Center Groningen, University of Groningen, Groningen, The Netherlands
4 Department of Gynecology and Obstetrics, Amsterdam University Medical Center, University of Amsterdam, Amsterdam, The Netherlands

5 Department of Pathology and Medical Biology, University Medical Center Groningen, University of Groningen, Groningen, The Netherlands

6 Groningen Kidney Center, Groningen, The Netherlands 